\title{
ZEITSCHRIFT FÜR REZENSIONEN ZUR GERMANISTISCHEN SPRACHWISSENSCHAFT
}

\author{
HERAUSGEBER \\ Alexander Lasch \\ Christa Dürscheid \\ Michael Elmentaler \\ Ulrike Freywald \\ Constanze Spieß
}

\section{DE GRUYTER}


ABSTRACTED/INDEXED IN Baidu Scholar · BDSL Bibliographie der deutschen Sprach- und Literaturwissenschaft · BLL Bibliographie Linguistischer Literatur · CNKI Scholar (China National Knowledge Infrastructure) $\cdot$ CNPIEC · Dimensions · DOAJ (Directory of Open Access Journals) · EBSCO (relevant databases) · EBSCO Discovery Service · ERIH PLUS (European Reference Index for the Humanities and Social Sciences) · Genamics JournalSeek · Google Scholar · IBR (International Bibliography of Reviews of Scholarly Literature in the Humanities and Social Sciences) · IBZ (International Bibliography of Periodical Literature in the Humanities and Social Sciences) · J-Gate * JournalTOCs · KESLI-NDSL (Korean National Discovery for Science Leaders) · Linguistic Bibliography · Microsoft Academic · MLA International Bibliography · Naviga (Softweco) · Norwegian Register for Scientific Journals, Series and Publishers $\cdot$ Primo Central (ExLibris) $\cdot$ Publons $\cdot$ ReadCube $\cdot$ Sherpa/RoMEO $\cdot$ Summon (Serials Solutions/ProQuest) · TDNet · Ulrich's Periodicals Directory/ ulrichsweb $\cdot$ WanFang Data $\cdot$ WorldCat (OCLC)

ISSN 1867-1691 · e-ISSN 1867-1705

Alle Informationen zur Zeitschrift, wie Hinweise für Autoren, Open Access, Bezugsbedingungen und Bestellformulare, sind online zu finden unter www.degruyter.com/zrs

REDAKTION Liane Drößler, Zeitschrift für Rezensionen zur germanistischen Sprachwissenschaft (ZRS), Technische Universität Dresden, Fakultät für Sprach-, Literatur- und Kulturwissenschaften, Institut für Germanistik, Professur für germanistische Linguistik und Sprachgeschichte, 01062 Dresden, Tel: +49 (0)351 463362 87, E-Mail: zrs-redaktion@mailbox.tu-dresden.de

JOURNAL MANAGER Anna Barkhoff, De Gruyter, Genthiner Straße 13, 10785 Berlin, Germany, Tel.: +49 (0)30 260 05-317, Fax: +49 (0)30 260 05-250, E-Mail: anna.barkhoff@ degruyter.com

ANZEIgenVERANTWORTLICHE Claudia Neumann, De Gruyter, Genthiner Straße 13, 10785 Berlin, Germany, Tel.: +49 (0)30 260 05-226, Fax: +49 (0)30 260 05-264,

E-Mail: anzeigen@degruyter.com

(C) 2018 Walter de Gruyter GmbH, Berlin/Boston

SATZ jürgen ullrich typosatz, Nördlingen

DRUCK Franz X. Stückle Druck und Verlag e.K., Ettenheim

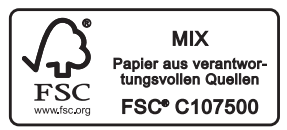




\section{Inhalt}

\section{Vorwort - 1}

Inger Petersen

Diana Gebele \& Alexandra L. Zepter (Hg.). 2016. Inklusion: Sprachdidaktische Perspektiven. Theorie, Empirie, Praxis (KöBeS - Kölner Beiträge zur Sprachdidaktik 11) -2

Thomas Krefeld

Helmuth Feilke \& Mathilde Hennig (Hg.). 2016. Zur Karriere von,Nähe und Distanz'. Rezeption und Diskussion des Koch-Oesterreicher-Modells (Germanistische Linguistik 306) -8

\section{Anna Volodina}

Henrike Helmer. 2016. Analepsen in der Interaktion. Semantische und sequenzielle Eigenschaften von Topik-Drop im gesprochenen Deutsch (OraLingua 13) Ewa Trutkowski. 2016. Topic Drop and Null Subjects in German (Linguistics and Philosophy 5) 15

Hans Jürgen Heringer/Toke Hoffmeister \& Verena Sauer (mit Stellungnahme von Wilhelm Köller)

Herausgeberkommentar zur Parallelrezension

Wilhelm Köller. 2016. Formen und Funktionen der Negation. Untersuchungen zu den Erscheinungsweisen einer Sprachuniversalie - 25

François Conrad

Hilke Elsen. 2016. Einführung in die Lautsymbolik - 45

Johannes Kabatek

Ekkehard Felder. 2016. Einführung in die Varietätenlinguistik (Germanistik Kompakt) -50

Konstanze Marx

Angelika Linke \& Juliane Schröter (Hg.). 2017. Sprache und Beziehung (Linguistik - Impulse \& Tendenzen) -55 


\section{Eckhard Meineke}

Christian Braun. 2017. Das Althochdeutsche aus textlinguistischer Sicht. Soziopragmatische Einordnung und ausgewählte textgrammatische Studien (Lingua Historica Germanica 14) — 61

Jörg Bücker

Torgrim Solstad. 2016. Lexikalische Semantik im Kontext: Die Spezifikation kausaler Relationen am Beispiel von durch - 66

\section{Franc Wagner}

Anna Andreeva. 2016. Metaphern im ethnischen Diskurs. Eine kognitiv-linguistische Analyse anhand der modernen deutschen Presse (Stauffenburg Linguistik 92) -73

\section{Peter Gallmann}

Thi Thu Hien Dang. 2016. Das Partizipialattribut im Deutschen zwischen System und Norm. Zur Systemkonformität von PII + habend (Germanistische Linguistik 304) -79

\section{Gianluca Cosentino}

Renate Freudenberg-Findeisen (Hg.). 2016. Auf dem Weg zu einer Textsortendidaktik. Linguistische Analysen und text(sorten)-didaktische Bausteine nicht nur für den fremdsprachlichen Deutschunterricht — 86

\section{Bettina M. Bock}

Heidrun Kämper \& Martin Wengeler (Hg.). 2017. Protest - Parteienschelte Politikverdrossenheit: Politikkritik in der Demokratie (Sprache - Politik - Gesellschaft 20) -91

\section{Luzius Thöny}

Oscar Eckhardt. 2016. Alemannisch im Churer Rheintal. Von der lokalen Variante zum Regionaldialekt (Zeitschrift für Dialektologie und Linguistik, Beihefte 162) -97

\section{Doris Stolberg}

Mark L. Louden. 2016. Pennsylvania Dutch. The Story of an American Language (Young Center Books in Anabaptist \& Pietist Studies) -104 


\section{Richard Schrodt}

Olga Heindl. 2017. Aspekt und Genitivobjekt. Eine kontrastiv-typologische Untersuchung zweier Phänomene der historischen germanischen Syntax (Stauffenburg Mediävistik 1) 110

Kristin Kuck

Ahmed Elgamel. 2017. Metaphorik in der politischen Pressesprache. Untersucht am Sprachenpaar Deutsch/Arabisch - 114

Magnus Breder Birkenes

Anna Cichosz, Jerzy Gaszewski \& Piotr Pęzik. 2016. Element Order in Old English and Old High German Translations (NOWELE Supplement Series) -120

Marlene Rummel

Silvia Bonacchi (Hg.). 2017. Verbale Aggression. Multidisziplinäre Zugänge zur verletzenden Macht der Sprache (Diskursmuster/Discourse Patterns 16) - 127

Horst M. Müller

Lars Bülow. 2017. Sprachdynamik im Lichte der Evolutionstheorie - Für ein integratives Sprachwandelmodell (Zeitschrift für Dialektologie und Linguistik Beihefte 166) 133

Wolfgang Imo

Elizabeth Couper-Kuhlen \& Margret Selting. 2018. Interactional Linguistics.

Studying Language in Social Interaction - 138

Martin Salzmann

Marek Konopka \& Angelika Wöllstein (Hg.). 2017. Grammatische Variation. Empirische Zugänge und theoretische Modellierung (Jahrbuch des Instituts für Deutsche Sprache 2016) - 145

\section{Gianluca Cosentino}

Katsiaryna Kanaplianik. 2016. Kognitionslinguistisch basierte Animationen für die deutschen Modalverben. Zusammenspiel der kognitiven Linguistik und des multimodalen Lernens bei der Sprachvermittlung (Transkulturelle Kommunikation 7) 152 


\section{Gerrit Kentner}

Jan Michalsky. 2017. Frageintonation im Deutschen. Zur intonatorischen Markierung von Interrogativität und Fragehaltigkeit (Linguistische Arbeiten 566) -155

\section{Markus Hundt}

Markus Denkler, Stephan Elspaß, Dagmar Hüpper \& Elvira Topalović (Hg.). 2017. Deutsch im 17. Jahrhundert. Studien zu Sprachkontakt, Sprachvariation und Sprachwandel. Gedenkschrift für Jürgen Macha. (Sprache - Literatur und Geschichte. Studien zur Linguistik/Germanistik 46) - 161

\section{Rüdiger Harnisch}

Nanna Fuhrhop, Renata Szczepaniak \& Karsten Schmidt (Hg.). 2017. Sichtbare und hörbare Morphologie — 167

\section{Karin Luttermann}

Jörg Roche. Unter Mitarbeit von Elisabetta Terrasi-Haufe, Martina Liedke, Mohcine Ait Ramdan und Mirjana Šimić. 2016. Deutschunterricht mit Flüchtlingen. Grundlagen und Konzepte (narr Studienbücher) — 174

\section{Sebastian Kürschner}

Peter Ernst \& Martina Werner (Hg.). 2016. Linguistische Pragmatik in historischen Bezügen (Lingua Historica Germanica 9) - 179

\section{Karina Frick}

Ágnes Veszelszki. 2017. Digilect. The Impact of Infocommunication Technology on Language (Studies in Information Science) - 185

\section{Nantke Pecht}

Stefaniya Ptashnyk, Ronny Beckert, Patrick Wolf-Farré \& Matthias Wolny (Hg.). 2016. Gegenwärtige Sprachkontakte im Kontext der Migration (Schriften des Europäischen Zentrums für Sprachwissenschaften (EZS) 5) — 191

Jorma Timo Huckauf und Netaya Lotze

Daniel Dejica, Gyde Hansen, Peter Sandrini \& Iulia Para. 2016. Language in the Digital Era. Challenges and Perspectives — 196

\section{Georg Albert}

Michael Beißwenger (Hg.). 2017. Empirische Erforschung internetbasierter Kommunikation. (Empirische Linguistik/Empirical Linguistics 9) - 202 


\section{Claus Ehrhardt}

Katharina Salzmann. 2017. Expansionen in der deutschen und italienischen Wissenschaftssprache. Kontrastive Korpusanalyse und sprachdidaktische Überlegungen (Studien Deutsch als Fremd- und Zweitsprache 3) — 208

Elisabeth Wellner

Andreas Lötscher. 2017. Areale Diversität und Sprachwandel im Dialektwortschatz (Zeitschrift für Dialektologie und Linguistik - Beiheft 169) - 214

Lars Bülow

Ekkehard Felder \& Friedemann Vogel (Hg.). 2017. Handbuch Sprache im Recht (Handbücher Sprachwissen 12) — 219

Monika Budde

Lutz Gunkel, Adriano Murelli, Susan Schlotthauer, Bernd Wiese und Gisela Zifonun. 2017. Grammatik des Deutschen im europäischen Vergleich. Das Nominal. Unter Mitarbeit von Christine Günther und Ursula Hoberg. Teilband 1: Funktionale Domänen, Wort und Wortklassen, Teilband 2: Nominalflexion, Nominale Syntagmen (Schriften des Instituts für Deutsche Sprache 14) - 224

Frank Liedtke

Peter Klotz. 2017. Modifizieren. Aspekte pragmatischer und sprachlicher Textgestaltung -231

Birte Kellermeier-Rehbein

Brigitte Ganswindt. 2017. Landschaftliches Hochdeutsch. Rekonstruktion der oralen Prestigevarietät im ausgehenden 19. Jahrhundert (Zeitschrift für Dialektologie und Linguistik - Beihefte 168) - 236

Tanja Ackermann

Rita Finkbeiner, Jörg Meibauer \& Heike Wiese (Hg.). 2016. Pejoration (Linguistik Aktuell / Linguistics Today 228) -241 
